\title{
Spectroscopic Orbit of the Eclipsing Binary BD $+52^{\circ} 2009$
}

\author{
B. Khalesseh ${ }^{1}$ \\ Department of Physics, University of Ferdowsi, Mashhad 91735-654, \\ Islamic Republic of Iran
}

\begin{abstract}
New radial velocity measurements of the Algol-type eclipsing binary $\mathrm{BD}+52^{\circ} 2009$, based on Reticon observations, are presented. The velocity measures are based on fitting theoretical profiles, generated by a physical model of the binary, to the observed cross-correlation function (ccf). Such profiles match this function very well, much better in fact than Gaussian profiles, which are generally used. Measuring the ccf's with Gaussian profiles yields the following results: $m_{p} \sin ^{3} i=$ $2.55 \pm 0.05 m_{\odot}, m_{s} \sin ^{3} i=1.14 \pm 0.03 m_{\odot},\left(a_{p}+a_{s}\right) \sin i=7.34 \pm 0.05 R_{\odot}$, and $m_{p} / m_{s}=2.23 \pm 0.05$. However, measuring the ccf's with theoretical profiles yields a mass ratio of 2.33 and following results: $m_{p} \sin ^{3} i=$ $2.84 \pm 0.05 m_{\odot}, m_{s} \sin ^{3} i=1.22 \pm 0.03 m_{\odot},\left(a_{p}+a_{s}\right) \sin i=7.56 \pm 0.05 R_{\odot}$. The system has a semi-detached configuration. By combining the solution of a previously published light curve with the spectroscopic orbit, one can obtain the following physical parameters: $m_{p}=2.99 m_{\odot}, m_{s}=1.28 m_{\odot},<$ $\left.T_{p}\right\rangle=9600 \mathrm{~K},\left\langle T_{s}\right\rangle=5400 \mathrm{~K},\left\langle R_{p}\right\rangle=2.35 R_{\odot},\left\langle R_{s}\right\rangle=2.12 R_{\odot}$. The system consists of an A0 primary and a G2 secondary.
\end{abstract}

\section{Introduction}

The Algol-type eclipsing binary $\mathrm{BD}+52^{\circ} 2009\left(\alpha=16^{h} 54^{m}, \delta=52^{\circ} 51^{\prime},(1900)\right.$, $\mathrm{A} 0$ + G2) has been the object of some photometric and spectroscopic studies. Light curves were published by Cester (1959), Mauder (1962), Winiarski (1971) and Mezzetti et al. (1980). Radial velocity curves were presented by Mauder (1962), Ebbighausen (1967) and Duerbeck \& Teuber (1978). Olson \& Weis (1974) pointed out some evidence of gas streams in the system and gave the secondary spectral type as F7. Duerbeck \& Teuber (1978) adopt F9. The reason for investigation is that in the Eighth Catalogue of the Orbital Elements of Spectroscopic Binary Systems (Batten, Fletcher, \& MacCarthy 1989), some elements of $\mathrm{BD}+52^{\circ} 2009$ are not determined, and this leaves some questions remaining about them. By detecting and measuring the radial velocities for both primary and secondary, one can derive the component masses and hence get a better view of the object. In fact the results show that cross-correlation is a reliable and fast method for measuring the radial velocities, particularly for the poor signals which are hard to detect. For some examples one can refer to

\footnotetext{
${ }^{1}$ Ed. note: this paper was substantially revised by C.D.S.
} 

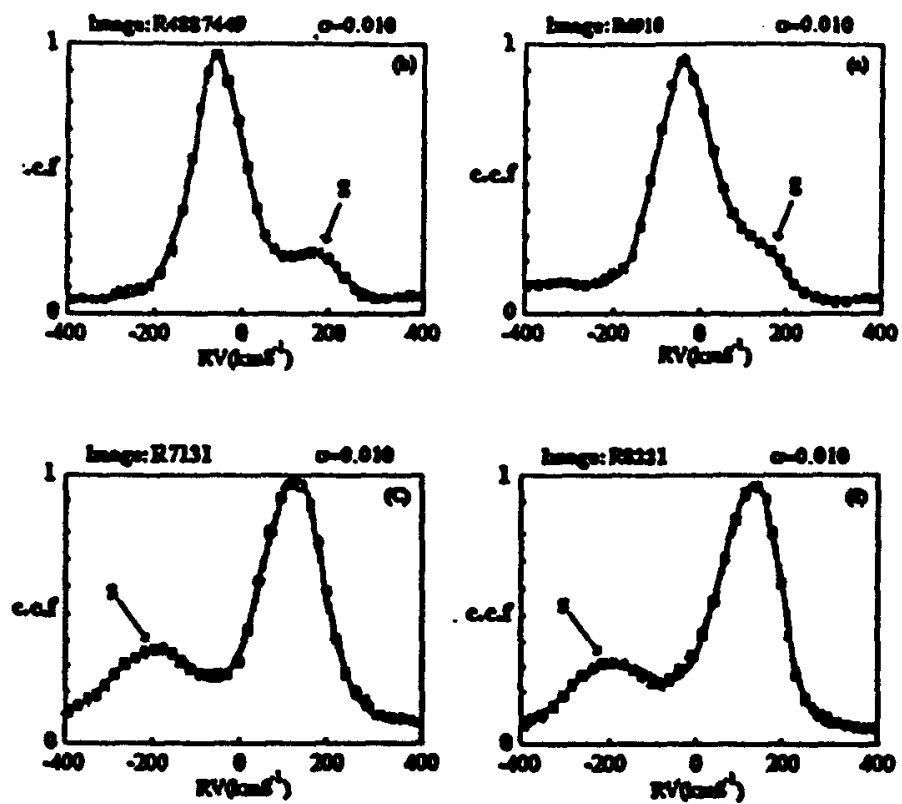

Figure 1. Sample Gaussian fits to some typical ccf's. A digital profile derived from an observed ccf of a spectrum is fitted to each curve. The secondary is detected in each case.

the papers by Hilditch, Hill, \& Khalesseh (1992), Ahn, Hill, \& Khalesseh (1992), Hill \& Khalesseh (1993), Hill, Perry, \& Khalesseh (1993), and Hill et al. (1994).

\section{Observations and reductions}

The observations discussed here were made between 1986 and 1987 with the Dominion Astrophysical Observatory's (DAO) coudé spectrograph on the 1.2 $\mathrm{m}$ telescope by Dr. Graham Hill of DAO. All were taken with an 1872-element Reticon array at a dispersion of $20 \AA \mathrm{mm}^{-1}$, and the exposures were limited to 30 $\min (0.012$ in phase) to minimize the effects of phase smearing. For all of these spectra the typical $\mathrm{S} / \mathrm{N}$ ratio is $>100: 1$ and the full-width at half-maximum (FWHM) of an arc line is $\sim 0.9 \AA$. The data were linearized in wavelength, $0.3 \AA$ / pixel (over the spectral range $\lambda \lambda 4000-4500 \AA$, and normalized to the continuum using the program REDUCE for use with VCROSS (Hilditch et al. 1992) in the cross-correlation process. Two stars were used as reference or comparison stars for the cross-correlation: Vega (HR 7001, A0V, $v \sin i=15 \mathrm{~km} \mathrm{~s}^{-1}$; RV $=-14$ $\mathrm{km} \mathrm{s}^{-1}$ ) for the A-type component and HD 154417 (F8.5 1V-V, RV $=-17 \mathrm{~km}$ $\mathrm{s}^{-1}$ ) for the presumed G-type subgiant. Both of these stars were successfully used by Khalesseh \& Hill (1992) with no problems with this choice. As it is not possible to measure a single cross-correlation function (ccf) for both velocities because of disparity in spectral types, the velocities were derived independently from ccf's generated by each reference star in turn. 


\section{RV measures}

\subsection{RV measures from Gaussian profiles}

The respective peaks were initially measured by fitting a Gaussian profile to each peak. The procedures followed here have been outlined in VCROSS and by Hill \& Khalesseh (1991). The radial velocities result from the fitting of Gaussian profiles to successive cross-correlations using Vega and then HD 154417 as standards. The ccf's were all right with both the standards, and this permits the weighting to be the same for both components. The rms's for the primary and secondary velocity measurements were 0.015 and 0.021 respectively. In Fig. $1(a, b, c, d)$ some samples of the Gaussian fit to some typical ccf's are shown and one can see the detection of the secondary.

\subsection{RV measures from synthetic profiles}

A series of synthetic line profiles from the binary model employed in the lightcurve solution can be generated, using the photometric data from Cester (1959) and the newly determined mass ratio. The procedure described by Hill, Fisher, \& Holmgren (1989) was followed. These profiles represent the shape of the ccf's better than the Gaussian ones, since they are hybrids, combining both rotational and Gaussian profiles. The synthetic profiles for each phase at which the spectra were available have been calculated. These profiles were used to fit the appropriate component of the ccf, for each comparison star in turn. The rms's for the primary and secondary velocity measurements were 0.015 and 0.017 respectively. The individual velocities are available from the author.

\section{Results}

\subsection{Orbit}

Orbital elements are given in Table 1 for the two types of measurement discussed above, and displayed in Fig. 2. One can see there are some systematic differences between the systemic velocities for the primary and secondary components. Since the secondary profiles are "buried" in the wings of the primary, it is not surprising that these velocities depend strongly on the profile used to model them.

\subsection{Photometric orbit}

Having established the mass ratio $\mathrm{q}=2.33$, one can derive some physical parameters for the system from the $\lambda 5200 \AA$ photoelectric observations of Cester (1959), using the Wilson-Devinney (1971) code. For the photometric solution, parameters were chosen according to the Straizys \& Kuriliene (1985) derivation to match the spectral type (B9 + F9 IV) given by Duerbeck \& Teuber (1978). The photometric solutions are given in Table 2 , in which the stellar radii presented are the means of $r_{\text {pole }}, r_{\text {side, }}$, and $r_{\text {back }}$, in units of the orbital radius. 
Table 1. Orbital Solutions for BD $+52^{\circ} 2009$

\begin{tabular}{|c|c|c|}
\hline $\begin{array}{l}\text { Orbit } \\
\text { Profile Type }\end{array}$ & $\begin{array}{c}\text { Non-Keplerian } \\
\text { Gaussian }\end{array}$ & $\begin{array}{l}\text { Keplerian } \\
\text { Synthetic }\end{array}$ \\
\hline Parameter & \multicolumn{2}{|c|}{ Primary } \\
\hline$V_{\circ}\left(\mathrm{km} \mathrm{s}^{-1}\right)$ & $-0.8 \pm 1.0$ & $-1.1 \pm 1.0$ \\
\hline$K_{\mathrm{p}}\left(\mathrm{km} \mathrm{s}^{-1}\right)$ & $95.9 \pm 1.3$ & $95.9 \pm 1.2$ \\
\hline$T_{\max }\left(\mathrm{JD}_{\odot}\right)($ fixed $)$ & 46523.336 & 46523.336 \\
\hline$P($ days $)$ (fixed) & 1.1988150 & 1.1988150 \\
\hline \multirow[t]{2}{*}{$\sigma^{2}\left(\mathrm{~km}^{2} \mathrm{~s}^{-2}\right)$} & 32.7 & 30.2 \\
\hline & \multicolumn{2}{|c|}{ Secondary } \\
\hline$V_{\circ}\left(\mathrm{km} \mathrm{s}^{-1}\right)$ & $5.2 \pm 2.3$ & $3.2 \pm 2.1$ \\
\hline$K_{\mathrm{s}}\left(\mathrm{km} \mathrm{s}^{-1}\right)$ & $213.9 \pm 2.7$ & $223.6 \pm 2.4$ \\
\hline$T_{\max }\left(\mathrm{JD}_{\odot}\right)($ fixed $)$ & 46522.737 & 46522.737 \\
\hline$P($ days)(fixed) & 1.1988150 & 1.1988150 \\
\hline \multirow[t]{2}{*}{$\sigma^{2}\left(\mathrm{~km}^{2} \mathrm{~s}^{-2}\right)$} & 148.1 & 118 \\
\hline & \multicolumn{2}{|c|}{ Combined } \\
\hline$V_{0}\left(\mathrm{~km} \mathrm{~s}^{-1}\right)$ & $2.0 \pm 1.5$ & $0.9 \pm 1.4$ \\
\hline$K_{\mathrm{p}}\left(\mathrm{km} \mathrm{s}^{-1}\right)$ & $95.9 \pm 2.1$ & $95.8 \pm 1.9$ \\
\hline$K_{\mathrm{s}}\left(\mathrm{km} \mathrm{s}^{-1}\right)$ & $213.9 \pm 1.7$ & $223.5 \pm 1.5$ \\
\hline$T_{\max }\left(\mathrm{JD}_{\odot}\right)($ fixed $)$ & 46523.336 & 46523.336 \\
\hline$P($ days $)$ (fixed $)$ & 1.1988150 & 1.1988150 \\
\hline \multirow[t]{2}{*}{$\sigma^{2}\left(\mathrm{~km}^{2} \mathrm{~s}^{-2}\right)$} & 95.1 & 75.9 \\
\hline & \multicolumn{2}{|c|}{ Derived Quantities } \\
\hline$m_{\mathrm{p}} \sin ^{3} i\left(m_{\odot}\right)$ & $2.55 \pm 0.05$ & $2.84 \pm 0.05$ \\
\hline$m_{\mathrm{s}} \sin ^{3} i\left(m_{\odot}\right)$ & $1.14 \pm 0.03$ & $1.22 \pm 0.03$ \\
\hline$a_{\mathrm{p}} \sin i\left(R_{\odot}\right)$ & $2.27 \pm 0.05$ & $2.27 \pm 0.05$ \\
\hline$a_{\mathbf{s}} \sin i\left(R_{\odot}\right)$ & $5.07 \pm 0.04$ & $5.29 \pm 0.04$ \\
\hline$q\left(m_{\mathrm{p}} / m_{\mathrm{s}}\right)$ & $2.23 \pm 0.05$ & $2.33 \pm 0.05$ \\
\hline
\end{tabular}

Table 2. Photometric Solution

\begin{tabular}{lc} 
Parameter & Value \\
\hline$<r_{\mathrm{h}}>$ & $0.343 \pm 0.004$ \\
$\left\langle r_{\mathrm{c}}>\right.$ & $0.268 \pm 0.006$ \\
$\left\langle T_{\mathrm{h}}>(K)\right.$ & $10400 \pm 190$ \\
$<T_{\mathrm{c}}>(K)$ & $6000 \pm 240$ \\
$q\left(m_{\mathrm{p}} / m_{\mathrm{s}}\right)$ & $2.33 \pm 0.05$ \\
$i\left(^{\circ}\right)$ & $79.0 \pm 0.3$ \\
\hline
\end{tabular}




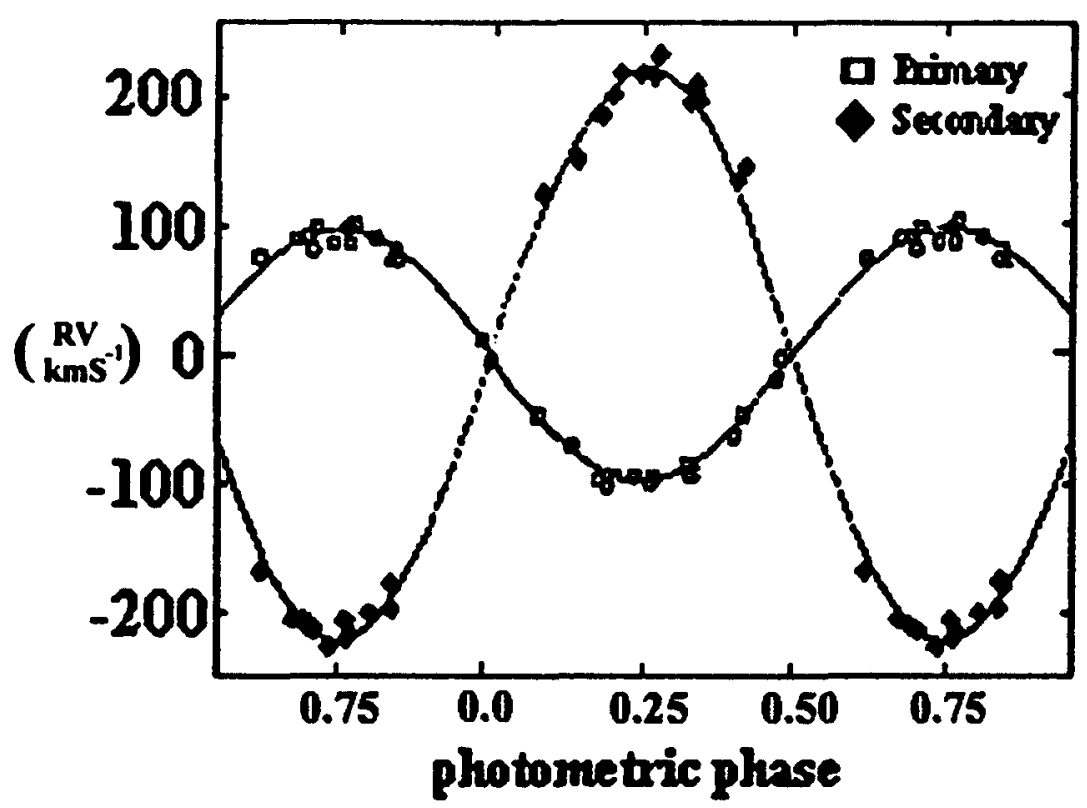

Figure 2. Velocity curve of $\mathrm{BD}+52^{\circ} 2009$ with reference to the photometric phase

Table 3. Physical parameters for $\mathrm{BD}+52^{\circ} 2009$

\begin{tabular}{lcc} 
Parameter & Primary & Secondary \\
\hline$m / m_{\odot}$ & $2.99 \pm 0.04$ & $1.28 \pm 0.03$ \\
$\left\langle R / R_{\odot}\right\rangle$ & $2.35 \pm 0.01$ & $2.12 \pm 0.01$ \\
$\left\langle T_{\odot}\right\rangle(K)$ & $9600 \pm 190$ & $5480 \pm 240$ \\
$\log m / m_{\odot}$ & $0.48 \pm 0.01$ & $0.11 \pm 0.01$ \\
$\log \left\langle R / R_{\odot}\right\rangle$ & $0.371 \pm 0.002$ & $0.326 \pm 0.002$ \\
$\log \left\langle T_{\odot}\right\rangle$ & $3.982 \pm 0.008$ & $3.739 \pm 0.018$ \\
$\log L / L_{\odot}$ & $1.62 \pm 0.02$ & $0.53 \pm 0.05$ \\
\multicolumn{2}{c}{ System } \\
$i\left(^{\circ}\right)$ & \multicolumn{2}{c}{$79.0 \pm 0.3$} \\
\hline \hline
\end{tabular}




\section{Discussion}

From the data in Tables 1 and 2 the physical parameters for the primary and secondary components were determined (see Table 3 ). The errors stem from the formal errors given in those tables, including the errors in mass ratio and an error in $\mathrm{T}_{r m e}$ (primary) of $190 \mathrm{~K}$. The position of BD $+52^{\circ} 2009$ in the massluminosity and mass-radius planes agrees well with data from Popper (1980) and Harmanec (1988). The period appears to have been constant since 1941, and the orbit is considered to be circular, because an elliptical solution shows the eccentricity not to be significant statistically.

Although it seems in the current investigation the secondary yields good velocities which lead to an acceptable mass ratio and a new photometric solution to model the system and its current evolutionary state, Mezzetti et al. (1980) have pointed out that the subgiant secondary is actually undersized and not in contact with its Roche lobe. To define an acceptable model for the system, further spectroscopic studies and theoretical calculations would be beneficial.

Acknowledgments. The author desires to express his gratitude to Dr. Graham Hill of DAO for permission to use his plate images. He also wishes to thank the DAO along with the Ferdowsi university for the use of their facilities. He also thanks Mr. Hessam Rahimi for his preparation of the manuscript.

\section{References}

Ahn, Y.S., Hill, G., \& Khalesseh, B. 1992, A\&A, 265, 597

Batten, A.H., Fletcher, J.M., \& MacCarthy, D.G. 1989, Publ. Dom. Astrophys. Obs., 17,1

Cester, B. 1959, Mem. Soc. Astron. Ital., 30, 287

Duerbeck, H.W., \& Teuber, D. 1978, Acta Astron. 28, 41

Ebbighausen, E.G. 1967, AJ, 72, 392

Harmanec, P. 1988, Bull. Astron. Inst. Czech. 39, 329

Hilditch, R.W., Hill, G., \& Khalesseh, B. 1992, MNRAS, 254, 82

Hill, G., Fisher, W.A., \& Holmgren, D. 1989, A\&A, 218, 152

Hill, G., Hilditch, R.W., Aikman, G.C.L., \& Khalesseh, B. 1994, A\&A, 276, 455

Hill, G., \& Khalesseh, B. 1991, A\&A, 245, 517

Hill, G., \& Khalesseh, B. 1993, A\&A, 276, 57

Hill, G., Perry, C.L., \& Khalesseh, B. 1993, A\&AS, 101, 579

Khalesseh, B., \& Hill, G. 1992, A\&A, 257, 199

Mauder, H. 1962, ZAp, 55, 59

Mezzetti, M., Cester, B., Giuricin, G., \& Mardirossian, R. 1980, A\&A, 39, 273

Olson, E.C., \& Weis, E.W. 1974, ApJ, 79, 642

Popper, D.M. 1980, ARA\&A, 18, 115

Straizys, V., \& Kuriliene, G. 1985, Ap\&SS, 80, 353

Wilson, R.E., \& Devinney, E.J. 1971, ApJ, 166, 605

Winiarski, M. 1971, Acta Astron., 21, 518 